\title{
Outcome Analysis of Major Cardiac Operations in Low Weight Neonates
}

\author{
Thierry Bové, MD, Katrien François, MD, Katya De Groote, MD, Bert Suys, MD, \\ Daniel De Wolf, MD, PhD, Hendrik Verhaaren, MD, PhD, Dirk Matthys, MD, PhD, \\ Annelies Moerman, MD, Jan Poelaert, MD, PhD, Piet Vanhaesebroeck, MD, PhD, and \\ Guido Van Nooten, MD, PhD
}

Departments of Cardiac Surgery, Pediatric Cardiology, Anaesthesiology, Cardiac Intensive Care, and Neonatology, University Hospital of Gent, Gent, Belgium

Background. From June 1995 to January 2003, 49 consecutive neonates of less than $2,500 \mathrm{~g}$ underwent early surgery for congenital heart disease. A retrospective analysis was performed to evaluate the early to medium term outcome.

Methods. Major cardiac surgery for congenital heart defects included a complete correction in 31 patients (group I) and a palliative procedure in 18 patients (group II). Mean age at operation was 15.2 days (1 day-90 days) and mean weight was $2,190 \mathrm{~g}(1,300 \mathrm{~g}-2,500 \mathrm{~g})$. Twentyfour children (49\%) were born prematurely. All neonates were critically ill and $47 \%$ were already ventilated preoperatively. Heart defects included mainly ventricular septal defect (10), tetralogy of Fallot complexes (8), aortic coarctation (8), transposition complexes (7), single ventricle anomalies (4), pulmonary atresia with intact septum (4), interrupted aortic arch (3), totally anomalous pulmonary venous return (3), and common atrioventricular septal defect (2).

Results. Overall surgical mortality was $18 \%$ : 4 neonates died after definitive repair and 5 after palliation; repre-

$\mathrm{T}^{\mathrm{h}}$ The New England Regional Infant Cardiac Program revealed clearly that children with congenital heart disease are more susceptible to have low birth weight, often associated to prematurity, and also that the presence of a cardiac malformation is alternately an important cause of death among these patients [1]. Low weight and premature age have both been recognized as risk factors for early mortality after cardiac surgery, supporting the perception that these particularly fragile babies are too small to withstand major surgery, including the eventual use of cardiopulmonary bypass [2,3].

However, the combination of a complex heart disorder and the functional immaturity of other vital organs makes the cardiorespiratory management of these children extremely difficult, presenting them often for a quest for surgery after weeks of insufficient or failed

\footnotetext{
Accepted for publication Dec 10, 2003.

Address reprint requests to Dr Bové, Department of Cardiac Surgery, U. Z. Gent, De Pintelaan 185 5K12, 9000 Gent, Belgium; e-mail: thierry.bove@ugent.be.
}

senting, respectively, $13 \%$ and $28 \%$ of each group. Postoperative morbidity occurred in half of the patients (53\%). Age, weight, prematurity, type of first surgical procedure, and use of cardiopulmonary bypass did not influence the early outcome. After a mean follow-up of 2.82 years ( 2 months to 6 years), survival was $87 \%$ in the correction group and $54 \%$ in the palliation group. All children were in NYHA class I-II. Freedom from reintervention at 18 months was $68 \%$ after correction versus $8 \%$ after palliation.

Conclusions. Cardiac surgery for congenital malformations in critically ill, low weight neonates can be achieved with acceptable mortality, at the cost of an increased morbidity. Early outcome seems independent of age, weight, prematurity, use of extracorporeal perfusion, and type of first intervention. Moreover, primary correction appears to result in an early survival benefit, remaining constant over time.

(Ann Thorac Surg 2004;78:181-7) (c) 2004 by The Society of Thoracic Surgeons medical therapy. Chang and colleagues [4] demonstrated that prolonging medical therapy to allow growth or weight gain did not result in improved survival, yielding thereby the eventual risk of death during medical management. Moreover, continuous progress in perfusion technology and perioperative treatment has encouraged the early repair of even complex heart anomalies, now achievable with good outcome. We reviewed our experience of an early surgical approach to these low weight neonates with severely symptomatic congenital heart disease and evaluated the short to intermediate term outcome after definitive repair and palliation.

\section{Material and Methods}

Patients

From June 1995 to January 2003, 49 infants with a weight of less than $2,500 \mathrm{~kg}$ underwent surgery for a simple or complex congenital cardiac disease. Early surgery represented a corrective or a palliative procedure, including 
Table 1. Contribution of the Congenital Heart Diseases

\begin{tabular}{lr}
\hline Ventricular septal defect (VSD) & 10 \\
With coarctation & 1 \\
Coarctation of aorta & 7 \\
Tetralogy of Fallot with/without pulmonary atresia & 8 \\
Transposition of great arteries & 3 \\
Simple & 4 \\
Complex with/without coarctation & 2 \\
Complete atrioventricular septal defect & 4 \\
With coarctation & 3 \\
Pulmonary atresia with intact septum & \\
Total anomalous pulmonary venous return & 1 \\
Interrupted aortic arch & 1 \\
With truncus arteriosus & 1 \\
With aortopulmonary window & \\
With subaortic and aortic stenosis & 1 \\
Single ventricle anomalies & 2 \\
Hypoplastic left heart disease & 1 \\
Tricuspid atresia and pulmonary stenosis & \\
Double inlet left ventricle and pulmonary stenosis & \\
\hline
\end{tabular}

the use of cardiopulmonary bypass when necessary. Neonates requiring ligation of an isolated patent ductus arteriosus were excluded.

The mean birth weight was $2,079 \pm 420 \mathrm{~g}$ (range, 1,000 to $2,500 \mathrm{~g}$ ) and raised to $2,192 \pm 340 \mathrm{~g}$ (range, 1,300 to $2,500 \mathrm{~g}$ ) at the time of surgery. Sixteen children weighed $2,000 \mathrm{~g}$ or less at operation. The mean gestational age was $35.1 \pm 4.06$ weeks (range, 27 to 40 weeks), and 24 patients $(49 \%)$ were born prematurely $(<37$ weeks of estimated gestational age). Mean age at operation was $15.2 \pm 18.9$ days (range, 1 to 90 days).

The distribution of congenital heart lesions are listed in Table 1. Noncardiac malformations included a chromosomal disorder in 6 patients (microdeletion 22q11 in 5, chromosome 17 deletion in 1), VACTERL association (affecting the vertebra, anus, cardiac system, trachea, esophagus, renal function, and limbs) in 1, digestive tract abnormalities as esophageal atresia in 4, and small intestinal atresia in 1, and hyaline membrane lung disease in 1. Previous noncardiac surgery comprised correction of the already mentioned digestive diseases.

Preoperatively all patients were severely ill: 26 (53\%) patients were in NYHA class III and $23(47 \%)$ in NYHA class IV. Correction of the metabolic and hemodynamic status required the use of intravenous prostaglandins for duct-dependent lesions, an atrial balloon septostomy in transposition complexes, and the eventual administration of inotropic agents and diuretics. Additionally, mechanical ventilation was initiated preoperatively in 23 patients $(47 \%)$.

\section{Operative Data}

Definitive complete correction (= group I) was performed in 31 infants, using median sternotomy and cardiopulmonary bypass in 23 . Standard neonatal cardiopulmonary bypass with aortic and bicaval venous cannulation included a Cobe roller pump and Dideco-Lilliput 1 membrane oxygenator (Cobe Cardiovasc. Inc., Arvada, CO) with minimal priming volume of $180 \mathrm{~mL}$, moderate hemodilution (hematocrit $25 \%$ to $30 \%$ ), and full-flow perfusion at moderate hypothermia of $25^{\circ} \mathrm{C}$. Cardiac arrest was induced by 15 to $20 \mathrm{~mL} / \mathrm{kg}$ antegrade cold crystalloid cardioplegia. Deep hypothermic circulatory arrest at $16^{\circ} \mathrm{C}$ was used in only 5 patients, with interrupted aortic arch and totally anomalous pulmonary venous return. Deep hypothermic circulatory arrest duration ranged from 10 to 43 minutes. The mean duration of cardiopulmonary bypass was $126 \pm 63$ minutes and the mean aortic cross-clamp time was $62 \pm 32$ minutes.

Coarctation repair by extended end-to-end anastomosis was achieved through lateral thoracotomy in 13 patients. In 8 children with isolated coarctation this procedure was considered as a complete definitive repair. One patient underwent first coarctation repair, followed by later elective closure of an unpalliated complete atrioventricular septal defect after 3 months. In 3 children coarctectomy was done as a first stage before complete correction of associated intracardiac disease. In 2 children coarctation surgery was combined to a pulmonary artery banding; both were included in the palliation group considering the residual noncorrected cardiac lesion.

In 18 children a palliative procedure was carried out (= group II). This consisted of a modified Blalock-Taussig shunt, placed through thoracotomy, in 14 patients with, respectively, tetralogy of Fallot $(n=3)$, pulmonary atresia with VSD $(n=4)$, pulmonary atresia with intact septum $(n=4)$, and cyanotic univentricular anomalies $(n=3)$. A central shunt of $3.5 \mathrm{~mm}$ was preferred in a 1,600 $\mathrm{g}$ weight neonate with pulmonary atresia and VSD because of the diminutive size of the pulmonary artery branches. The size of the Blalock-Taussig shunts varied from $4 \mathrm{~mm}(\mathrm{n}=$ 7) to $5 \mathrm{~mm}(\mathrm{n}=7)$.

A pulmonary artery banding was performed, simultaneously with coarctation repair, for a complete atrioventricular canal in an infant of 1,300 $\mathrm{g}$ with intractable heart failure, and for a complex double outlet right ventricle type ventricular septal defect with transposition of the great arteries in 1 patient with associated hypoplasia of the left lung. Finally, a Norwood I procedure was considered as a palliative procedure including the use of extracorporeal circulation, in 1 neonate with hypoplastic left heart disease.

\section{Data Collection and Analysis}

Perioperative data were obtained through retrospective review of hospital records. Outcome analysis included early mortality (defined as death during postoperative hospitalization) and morbidity, focused on incidence and type of complications, duration of ventilation and inotropic support, intensive care, and hospital stay. Crosssectional follow-up, including clinical status, weight evolution, psychomotor development, reintervention rate, and late survival, was available for all patients.

Data are expressed as mean values, standard deviations, and range. Comparison of categorical variables between two groups was performed with $\chi^{2}$ analysis. Continuous variables were compared by the paired or 
unpaired samples $t$ test. A $p$ value of less than 0.05 was considered statistically significant. Actuarial survival and freedom of reintervention analysis was done with the Kaplan-Meier product limit method and log-rank evaluation. Statistical processes were conducted with the SPSS 11.0 for Windows package (SPSS Inc., Chicago, IL).

\section{Results}

\section{Demographic Data}

The demographic data and preoperative status of the overall group of low weight neonates have already been described above. Depending on the type of operation, two groups could be identified: a definitive correction group (I) and a palliative procedure group (II). Comparing their age at operation $(20.3 \pm 21.7$ vs $6.5 \pm 7.1$ days, $p$ $=0.02)$, weight at operation ( $2.2 \pm 0.3$ vs $2.2 \pm 0.4 \mathrm{~kg}, p=$ $0.76)$, and premature state $(19 / 31=61 \%$ vs $6 / 18=33 \%, p$ $=0.06$ ), the neonates in whom a corrective operation was performed, were older despite the higher occurrence of prematurity. However, regarding the preoperative clinical status, both groups were equally ill, with ventilation need in 14 of $31(45 \%)$ patients of group I and in 9 of 18 $(50 \%)$ patients of group II $(p=0.74)$. The average duration of preoperative ventilation was $4.1 \pm 3.5$ days $(4.9 \pm$ 4.1 day in group I vs $2.7 \pm 1.9$ days in group II, $p=0.15$ ).

The age difference between both groups is specifically disease related since preoperative hemodynamic stabilization is obtained more easily and more constantly by adequate medical treatment for ventricular septal defect or by a percutaneous atrial septostomy for transposition complexes. Moreover, medical therapy was first attempted in neonates presenting only a ventricular septal defect as a primary cause of clinical deterioration.

\section{Early Outcome}

HOSPITAL MORTALITY. Overall, there were 9 early deaths $(18 \%)$; ie, $4(13 \%)$ after definitive correction and $5(28 \%)$ after palliation. In the first group, all four patients died immediately perioperatively, due to cardiac failure $(\mathrm{n}=$ 2 , one by myocardial infarction after arterial switch operation, one after correction of interrupted aortic arch and Ross-Konno procedure), intractable pulmonary hypertension in one patient with interrupted aortic arch and truncus arteriosus repair, and technically incorrectable diffuse pulmonary venous stenoses $(n=1)$. These deaths correlated to a longer cardiopulmonary bypass time (105.8 \pm 43 vs $226.3 \pm 43.9$ minutes, $p=0.006)$ and aortic cross-clamp time $(55.5 \pm 29.7$ vs $92.5 \pm 27.5$ minutes, $p=0.06$ ). In particular, coarctation repair was done without mortality.

Causes of death after palliative surgery were sepsis (n $=3)$, cerebral hemorrhage $(\mathrm{n}=1)$, and low cardiac output and hypoxemia after a Norwood I procedure $(\mathrm{n}=$ 1). Except for the patient with hypoplastic left heart disease, these deaths occurred two to fourteen days postoperatively. Analysis of risk factors for early death revealed that age, weight, prematurity, type of first sur-
Table 2. Risk Factor Analysis for Early Mortality

\begin{tabular}{lc}
\hline Risk Factors & $p$ Value \\
\hline Age & 0.19 \\
Weight & 0.57 \\
Prematurity & 0.66 \\
Procedure type & 0.19 \\
Use of CPB & 0.66 \\
\hline
\end{tabular}

gical procedure, and use of cardiopulmonary bypass did not significantly influence the early outcome (Table 2).

\section{Early Morbidity}

Minor to major complications were noticed in 26 (53\%) patients, without significant differences between both types of operations (17/31 [55\%] in group I vs 8/18 [44\%] in group II, $p=0.62$ ). This included pulmonary problems such as pneumonia and lobe atelectasis $(n=13)$, low cardiac output $(\mathrm{n}=8)$, arrhythmias as atrioventricular block $(n=2)$ or junctional ectopic tachycardia $(n=2)$, renal failure necessitating peritoneal dialysis $(n=2)$, cerebral hemorrhage $(n=2)$, and severe sepsis due to pulmonary infection $(n=3)$ and to enterocolitis $(n=1)$. Cardiorespiratory complications appeared more frequently after definitive correction (17/31 [55\%] in group I vs $6 / 18[33 \%]$ in group II, $p=0.14)$. However, neurologic damage, often feared in relation to the use of extracorporeal perfusion in premature infants, appeared in one patient of each group: one patient after coarctation repair with partial recovery and one patient after a BlalockTaussig shunt with fatal outcome.

Early reoperation was required in 5 patients; in 2 after corrective repair versus 3 after palliation. One child with Shone"s syndrome who had uncomplicated coarctation surgery, underwent a Ross-Konno procedure after unsuccessful balloon dilatation of a complex aortic valve stenosis. Another neonate developed early symptomatic recoarctation and needed surgical revision after 1 week. Reintervention after palliative surgery was needed for early stenosis of a shunt $(\mathrm{n}=1)$, diaphragm paralysis ( $=1$ ), and recurrent atelectasis due to partial upper lobe torsion around a Blalock-Taussig shunt $(n=1)$.

The postoperative ventilation time (median, 48 hours [range, 6 to 336 hours] in group I vs 18 hours [range, 4 to 408 hours] in group II, $p=0.50$ ) and inotropic support duration (median, 36 hours [range, 0 to 172 hours] in group I vs 10 hours [range, 0 to 336 hours] in group II, $p$ $=0.54$ ) were comparable. Also, intensive care stay (median, 5 days [range, 1 to 32 days] in group I vs 3 days [range, 1 to 43 days] in group II, $p=0.90$ ) and hospital stay (median, 15 days [range, 5 to 86 days] in group I vs 12 days [range, 6 to 55 days] in group II, $p=0.98$ ) appeared to be independent of the type of surgical procedure.

\section{Late Outcome}

Complete follow-up was available for all 40 survivors over an average period of $2.82 \pm 2.51$ years, and comprised clinical evolution, late reintervention rate, and survival. 


\section{Clinical Assessment}

The majority of the surviving neonates were clinically doing well after early operative management of their congenital heart disorder with 31 children in NYHA class I, and 7 children in NYHA class II. Two infants remained in NYHA class III. Regarding the clinical neurodevelopmental state, obvious mental and motor retardation was observed in 4 children, of whom 2 were born prematurely. One had associated CATCH 22 chromosomal disorder and another suffered from cerebral hemorrhagic infarction after coarctation repair. Additionally, the weight evolution during the first year after primary corrective repair as well as after palliation, showed a growth pattern similar to that of low birth weight infants not affected by a congenital heart disease (Figs $1 A$ and $1 B)$.

\section{Late Reinterventions}

Overall, 18 of the 40 survivors underwent a total number of 20 reinterventions, after a mean delay of $8.6 \pm 5.3$ months. Late operation as the definitive repair of the cardiac anomaly was mandatory in 11 of the 13 children treated initially by palliation, resulting in two late deaths. Seven infants needed reintervention after definitive correction, respectively, by a percutaneous procedure in 5 and by a surgical method in 2 . The interventional technique consisted of dilatation of recoarctation $(n=2)$, aortic valve dilatation $(n=1)$, and relief of a pulmonary branch stenosis $(n=2)$. Late surgery in this group was required for recurrent anastomotic stenosis after total anomalous pulmonary venous drainage repair and for definitive correction of a nonpalliated common atrioventricular canal, secondary to previous coarctation repair. Both latter procedures were performed without mortality. Actuarial freedom from reintervention was $68 \%$ at 36 months in children undergoing definitive correction at neonatal age, versus $8 \%$ at 18 months in those treated by a palliative procedure, which was statistically significant $(p=0.002)($ Fig 2$)$.

\section{Late Survival}

Late survival was $76 \%$ within a follow-up duration of 2 months to 6 years. Regarding the operative procedure, actuarial survival at 1 year and beyond was $87 \%$ for the definitive correction group and $54 \%$ for the palliation group ( $p=0.02)$ (Fig 3 ).

In group I survival remained steady over time. But, in group II, there were 3 late deaths after neonatal palliation. One child died at the age of 5 months, after correction of a palliated common atrioventricular septal defect, and another child died due to severe residual systemic valve regurgitation after a Hemifontan palliation of a single ventricle anomaly. Finally, a third child, with palliated double outlet right ventricle and transposition of the great vessels, was lost at the age of 3 months and before complete repair, because of recurrent respiratory insufficiency in the presence of left lung hypoplasia.

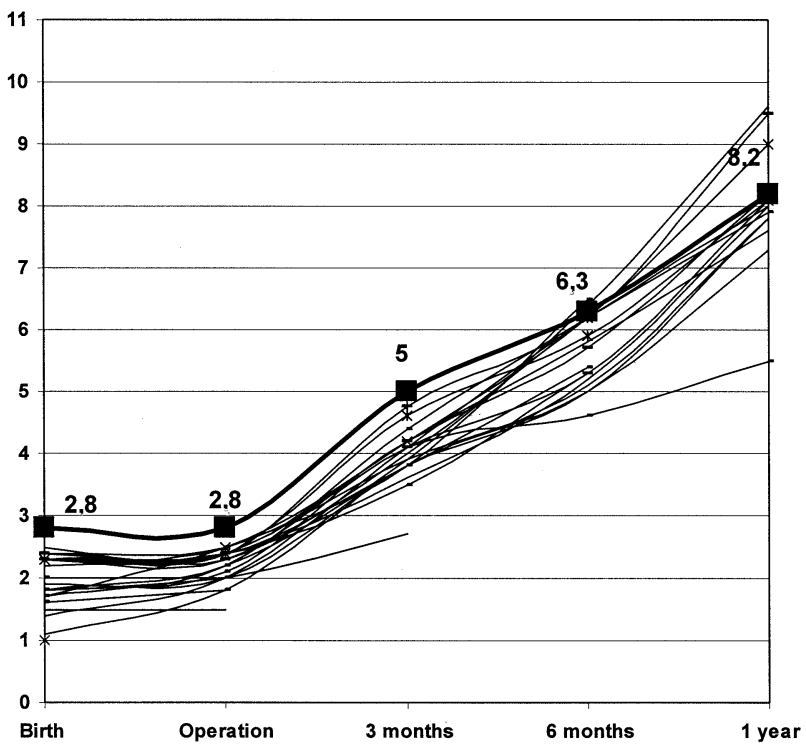

A

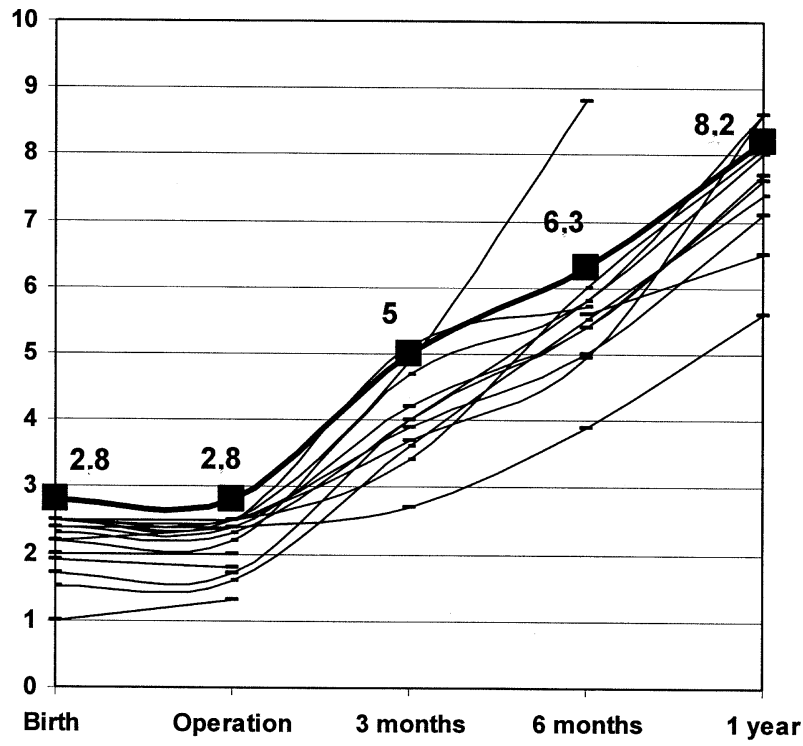

B

Fig 1. (A) Weight evolution after corrective surgery. (B) Weight evolution after palliative surgery.

\section{Comment}

The knowledge that early restoration of a normal cardiovascular physiology has a beneficial effect on the postnatal maturation of major organs such as lungs, brain, and heart, advanced gradually the surgical correction of various congenital heart diseases towards the neonatal age. However, the encouraging results of neonatal cardiac surgery can be compromised by some limiting factors.

Previous reports have suggested that low weight and premature age are risk factors for poor outcome after correction of congenital heart defects [2, 3]. Additionally, the perception that these premature infants are more 


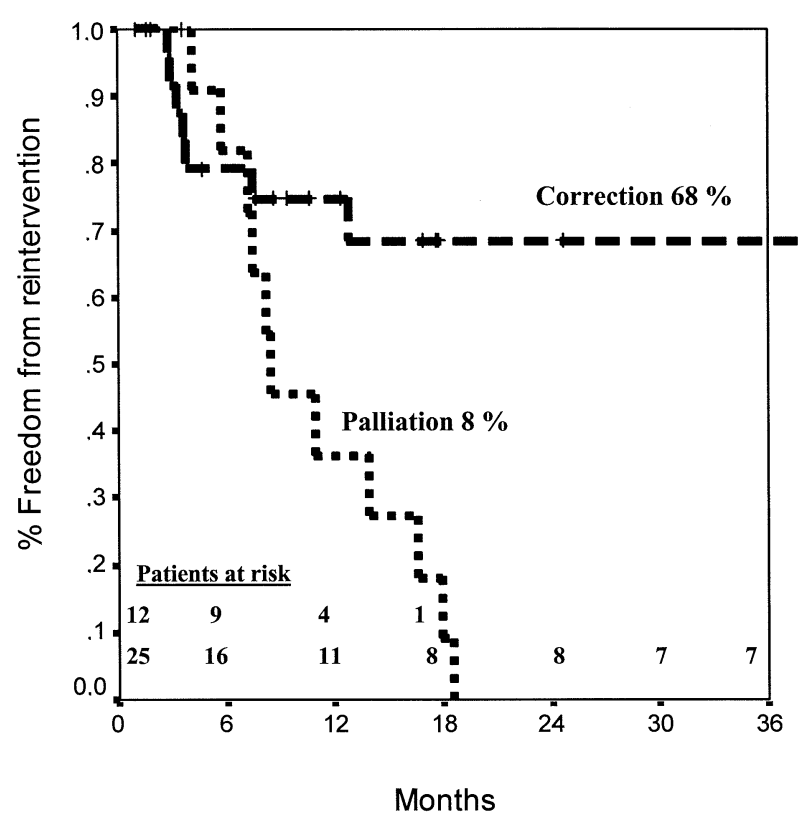

Fig 2. Actuarial freedom from reintervention.

vulnerable by associated issues such as immature lungs and the potential risk for cerebral hemorrhage, has supported the belief of many neonatologists that corrective surgery with the use of cardiopulmonary bypass has to be avoided in favor of a supportive medical therapy or surgical palliation.

However, in a study of 100 low birth weight neonates, Chang and colleagues [4] concluded that deferring an early intervention to promote weight gain is not recommended since the outcome of a prolonged medical therapy does not exceed that of early surgery. Moreover,

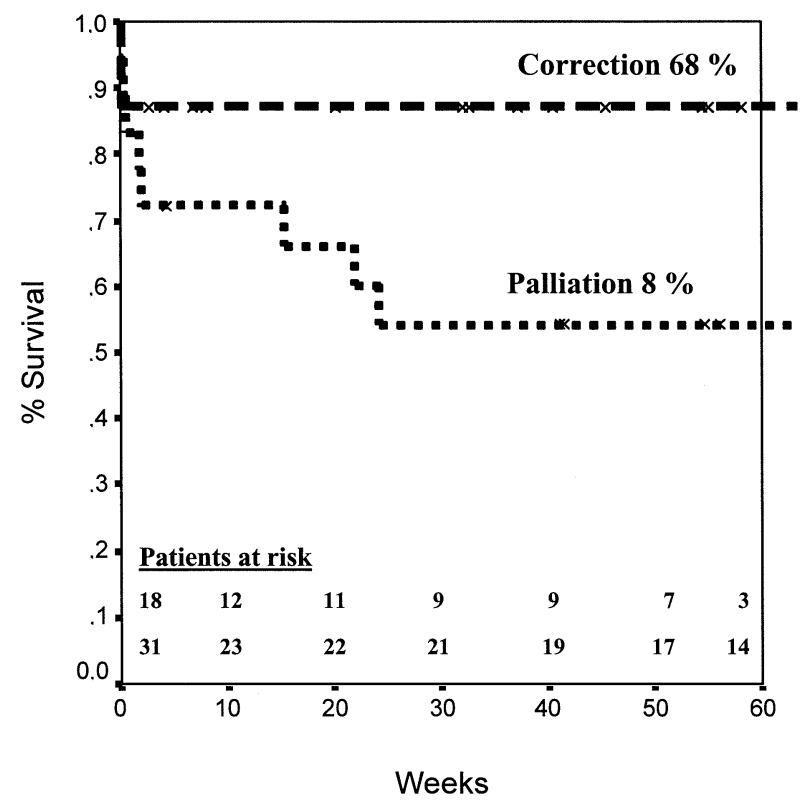

Fig 3. Actuarial survival curve after correction versus palliation. focusing particularly on this low weight neonatal population, some groups reported recently that a complete repair of even complex lesions could be achieved with an acceptable mortality of $10 \%$ to $22 \%$ [5-7].

Our study involves a consecutive series of low weight neonates undergoing early surgical management, either by complete correction or by palliation. We report a $13 \%$ mortality rate after definitive repair and found that the use of cardiopulmonary bypass was not associated with an increased mortality, compared to palliative procedures. However, the application of extracorporeal circulation enhances the cardiac and pulmonary morbidity, resulting in a more frequent occurrence of reversible low cardiac output state, arrhythmias, and pulmonary complications.

According to Reddy and colleagues [5], cardiopulmonary bypass time and aortic cross-clamp time are longer for those patients who died. Both are not considered as risk factors for early mortality per se, but rather reflect a higher complexity of the malformation, leading to a technically more demanding repair and subsequently prolonged circulatory support.

Deep hypothermic circulatory arrest is more frequently used in these very small babies, because of technical issues and a more convenient operating field. Presently, the application of alternative perfusion techniques has allowed avoidance of the systematic use of circulatory arrest and thereby decreases the substantial risk of neurologic sequellae [8]. In this series, circulatory arrest was only used for repair of interrupted aortic arch and associated anomalies, and intermittently for repair of anomalous pulmonary venous return, and did not result in adverse neurologic outcome.

Within this patient selection are included the neonates presenting aortic coarctation as an isolated disease or in combination with more complex anomalies. Operative correction was achieved without mortality, concurring favorably with the recently reported data (Bacha and colleagues [9]) on coarctation repair in infants weighing less than $2 \mathrm{~kg}$. Of course, the early outcome of corrective procedures is undeniably diluted by the excellent results of coarctation repair in this population. Even after exclusion of these definitive operations done without the use of cardiopulmonary bypass, mortality rises slightly to $17 \%$. These results encourage our policy of a two-stage approach with first coarctation repair through thoracotomy, rapidly followed by correction of the intracardiac anomalies through sternotomy. This strategy obviates the need for longer cardiopulmonary bypass time with the eventual use of deep hypothermic circulatory arrest when correction of aortic coarctation is aimed simultaneously through an anterior approach. Otherwise, early restenosis is another concern in these small infants [9], and occurred in three of our patients. This could be managed effectively by balloon dilatation in two cases. As mentioned by the same group [9], it can sometimes be difficult to deal with severe symptomatic residual coarctation in these neonates and early surgical rerepair must then be considered, which we had to do in one patient.

Although it is well known that the premature neonatal 
heart is less compliant to volume overload, as induced by a systemic-pulmonary artery shunt [10], some congenital heart defects are not amenable to primary repair, due to anatomical features. Some authors demonstrated that performing a palliative operation in these low weight babies might not necessarily decrease the risk for mortality, especially in disorders where a single ventricle has to support the systemic and the pulmonary circulation [6]. In our group, mortality after a palliative procedure is $28 \%$. Only one death was related to a univentricular physiologic disorder, namely a hypoplastic left heart disease. However, determining the Norwood I operation as a palliative or corrective procedure in this setting is debatable, knowing the possible difficulties for systemic to pulmonary blood flow equilibration after the use of a longstanding cardiopulmonary bypass. In contrast to the early deaths after definitive repair, most deaths after palliation occurred later on during the postoperative course, often secondary to a progressive imbalance of both circulations promoted by sepsis of pulmonary or intestinal origin. The optimal shunt size to achieve a stable acute and chronic systemic-pulmonary blood flow ratio is not clearly defined. Even if a direct correlation between morbidity and mortality and the use of a larger shunt was not apparent in this series, a 4-mm shunt seems more appropriate in these small children.

Our palliated patient group comprised eight patients with tetralogy of Fallot with and without pulmonary atresia, which is possibly repairable in the presence of a favorable pulmonary artery arborization. Excellent results of neonatal correction of this entity have already been reported, even in low weight neonates, but at the cost of a more frequent use of transannular patch repair of the right ventricular outflow tract, the risk of incomplete relief of distal pulmonary artery stenosis, and the eventual need of a pulmonary conduit implantation [11, 12]. We preferred a more conservative approach by treating these small and severely symptomatic patients by initial palliation, followed by complete repair during the first year of life. Whether this policy is justified in comparison to primary neonatal correction in terms of survival and reoperation rate requires further investigation.

It is logical to assume that restoration of cardiovascular physiology should normalize the clinical status and growth of these children. Analysis of the weight evolution during the first postoperative year shows that growth evolution is similar to that seen in low weight neonates without cardiac disease. This observation is independent of the applied type of surgical procedure, and correlates with other series [5]. Moreover, although this particular patient population is at risk for developmental impairment by the low weight itself, the cardiac disorder, the often associated syndromes, and the early surgery including the eventual use of extracorporeal perfusion, the clinical neuropsychological evolution in this series seems acceptable with only one procedure related event. However, the application of more refined neurodevelopmental scales could, perhaps, objectivate more appropriately psychomotor sequellae as has been reported by Dittrich and colleagues [13] in a prospective assessment of one-year-old children after surgery for congenital heart disorders.

Addressing the issue of late reinterventions, it is obvious that a later reoperation is mandatory in those infants who were initially managed by a palliative procedure, entailing a potential risk of late operative mortality. We had three late deaths in this group, two of which were surgery related. When early complete repair was achievable, Reddy and colleagues [5] reported that the rate of reintervention was comparable to that of operations performed on term neonates. In our study the late operation rate was $26 \%$ after definitive correction and could be managed by a less invasive, interventional catheter-based procedure in five of the seven infants, without mortality. These late observations finally result in a significant difference in late survival between the definitive repair group and the palliation group. But more interesting is the finding that the survival after initial corrective surgery remained constant, once these children had undergone their first operation successfully.

However, this study carries the limitations related to its retrospective character and the large heterogeneity of the involved congenital heart defects. Dividing this patient population in groups according to the primary surgical procedure, correction versus palliation, troubles undoubtedly the interpretation of the results. The nature of the cardiac disease can be such that effective palliation is nonexistent (eg, interrupted aortic arch) or that specific anatomical barriers exclude primary definitive repair (eg, single ventricle hearts). Also, the policy to treat some specific diseases preferentially in a staged fashion may further bias the final conclusions. From this point of view, concluding that primary definitive correction should be pursued because of the advantage on survival might be too simple. Even if one intuitively believes that the risk of early surgery is promoted by the prematurity and low weight, compared to a normal neonatal population, it seems to us that rather the complexity of the cardiac disease and its secondary physiologic disturbance are more relevant to the outcome. This is emphasized by the fact that age, weight, prematurity, type of initial operative procedure, and use of cardiopulmonary bypass were not identified as risk factors for early death.

Based on these results, we feel encouraged that an early surgical approach in this particular subset of low weight neonates with critical heart disease can be performed with acceptable early outcome, but at the cost of considerable morbidity. In the presence of favorable anatomical features, the low weight and prematurity per se should not preclude the attempt for complete correction with the use of cardiopulmonary bypass, in favor of a prolonged medical therapy or eventual palliative procedure. Moreover, successful primary complete repair of even complex malformations appears to result in an early survival benefit that remains constant over time.

\section{References}

1. Fyler D. Report of the New England Regional Infant Cardiac Program. Pediatrics 1980;65:377-461. 
2. Kirklin JK, Blackstone EH, Kirklin JW, Mc Kay R, Pacifico $\mathrm{AD}$, Bageron LM. Intracardiac surgery in infants under age 3 months: incremental risk factors for hospital mortality. Am J Cardiol 1981;48:500-6.

3. Pawade A, Waterston K, Laussen P, Karl TR, Mee RBB. Cardiopulmonary bypass in neonates weighing less than 2.5 $\mathrm{kg}$ : analysis of risk factors for early and late mortality. J Card Surg 1993;8:1-8.

4. Chang AC, Hanley FL, Lock JE, Castaneda AR, Wessel DL. Management and outcome of low birth weight neonates with congenital heart disease. J Pediatr 1994;124:461-6.

5. Reddy MV, McElhinney DB, Sagrado T, Parry AJ, Teitel DF, Hanley FL. Results of 102 cases of complete repair of congenital heart defects in patients weighing 700 to 2500 grams. J Thorac Cardiovasc Surg 1999;117:324-31.

6. Rossi AF, Seiden HS, Sadeghi AM, et al. The outcome of cardiac operations in infants weighing two kilograms or less. J Thorac Cardiovasc Surg 1998;116:28-35.

7. Beyens T, Biarent D, Bouton JM, et al. Cardiac surgery with extracorporeal circulation in 23 infants weighing $2500 \mathrm{~g}$ or less: short to intermediate outcome. Eur J Cardiothorac Surg 1998;14:165-72.

8. Reddy MV, Hanley FL. Techniques to avoid circulatory arrest in neonates undergoing repair of complex heart defects. Ped Card Surg Annual 2001;4:277-80.

9. Bacha EA, Almodovar M, Wessel DL, et al. Surgery for coarctation of the aorta in infants less than $2 \mathrm{~kg}$. Ann Thorac Surg 2001;71:1260-4.

10. Friedman WF. The intrinsic physiological properties of the developing heart. Prog Cardiovasc Dis 1972;15:87-111.

11. Di Donato RM, Jonas RA, Lang P, Rome JJ, Mayer JE, Castaneda AR. Neonatal repair of tetralogy of Fallot with and without pulmonary atresia. J Thorac Cardiovasc Surg 1991;101:126-37.

12. Hennein HA, Mosca RS, Urcelay G, Crowley DC, Bove EL. Intermediate results after complete repair of tetralogy of Fallot in neonates. J Thorac Cardiovasc Surg 1995;109:332-44.

13. Dittrich H, Buhrer C, Grimmer I, Dittrich S, Abdul-Khaliq H, Lange PE. Neurodevelopment at 1 year of age in infants with congenital heart disease. Heart 2003;89:436-41.

\section{Southern Thoracic Surgical Association: Fifty-first Annual Meeting}

The Fifty-first Annual Meeting of the Southern Thoracic Surgical Association (STSA) will be held November 4-6, 2004, in Cancun, Mexico.

The Postgraduate Course will be held the morning of Thursday, November 4, 2004, and will provide in-depth coverage of thoracic surgical topics selected primarily as a means to enhance and broaden the knowledge of practicing thoracic and cardiac surgeons.

Manuscripts accepted for the Resident Competition need to be submitted to the STSA headquarters office no later than September 17, 2004. The Resident Award will be based on abstract, presentation, and manuscript.

Applications for membership should be completed by September 15, 2004, and forwarded to John P. Marbarger,
MD, Membership Committee Chairman, Southern Thoracic Surgical Association, 633 N Saint Clair St, Suite 2320, Chicago, IL 60611-3658.

John H. Calhoon, MD

\section{Secretary/Treasurer}

Southern Thoracic Surgical Association

633 N Saint Clair St, Suite 2320

Chicago, IL 60611-3658

(800) 685-STSA or (312) 202-5892

fax: (312) 202-5801

e-mail: stsa@stsa.org; website: http://www.stsa.org. 